\title{
Evolving concepts in muscle-invasive bladder cancer
}

\author{
Peter C. Black ${ }^{1} \cdot$ Wassim Kassouf $^{2}$
}

Published online: 7 January 2016

(C) Springer-Verlag Berlin Heidelberg 2016

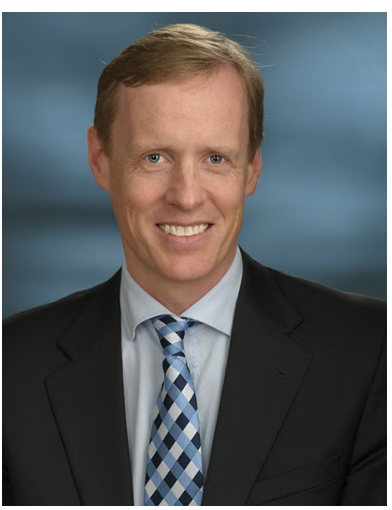

Peter C. Black

Advances in the treatment of muscle-invasive bladder cancer (MIBC) are occurring on multiple fronts. While most improvements are incremental, critical large clinical trials and potential major breakthroughs are on the horizon.

In this issue of the Journal, VanderWalde et al. highlight the growing recognition that geriatric oncology is an essential component of caring for patients with MIBC [1]. Age is a particularly important factor in MIBC because the median age of bladder cancer patients is higher than for any other cancer, and only approximately half of patients

Peter C. Black

pblack@mail.ubc.ca

Wassim Kassouf

wassim.kassouf@muhc.mcgill.ca

1 Department of Urologic Sciences, Vancouver Prostate Centre, University of British Columbia, Level 6, 2775 Laurel St, Vancouver, BC V5Z 1M9, Canada

2 Department of Surgery (Urology), McGill University Health Center, 1001 Decarie Blvd, D02.7210, Montreal, QC H4A 3J1, Canada

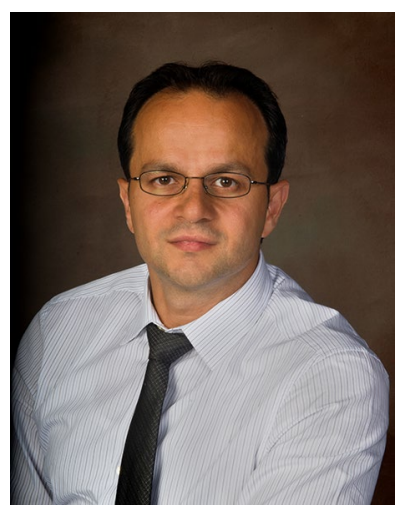

Wassim Kassouf

with MIBC are receiving treatment with curative intent [2]. The need for geriatric assessment of the MIBC patient and the involvement of a geriatrician in the multidisciplinary management of these patients go well beyond simple fine-tuning of therapy and instead are critical to increasing access to aggressive therapy, which should not be ruled out based on age alone. Various preoperative prehabilitation programs integrating exercise and nutrition will likely play a significant role in the future, in particular with the elderly and frail who may be rendered fitter for radical interventions.

Patient age also influences the choice of urinary diversion at the time of radical cystectomy, with widely varying attitudes on the appropriateness of continent diversion in older patients. Hugen and Daneshmand make the case that age itself should not be the main determinant of whether a patient receives continent diversion [3]. To some degree, continent diversion is the default option in their hands, and the ileal loop is offered only for specific contraindications. Siddiqui and Izawa, on the other, believe that the potential quality-of-life advantages of the continent diversion do 
not justify the high risk of complications and a potentially slower recover compared to the ileal conduit in elderly patients [4]. While there is no right answer in this debate, it should be clear again that age alone should not be an exclusion criterium.

The surgical management of MIBC is evolving with advances in robotic surgery. Patel et al. make the case for robotic cystectomy being the new standard of care [5], while Kiss et al. maintain that the open surgery remains the gold standard [6]. Robotic prostatectomy has become the standard in many parts of the world despite the absence of high-level evidence supporting this transition. The MIBC community has attempted to use prospective randomized trials to guide this evolution, but the widespread adoption of the robot for radical cystectomy has taken firm hold despite trials demonstrating little apparent benefit with respect to complication rates and postoperative recovery. However, with the urinary diversions now also being performed intracorporeally, further comparative studies are required. Furthermore, a more philosophical question that needs to be addressed is whether the urologic community should set the target for robotic cystectomy with intracorporeal diversion to be equivalent or rather superior to the open approach in order for it to become the standard intervention.

The biggest potential advance on the horizon in the surgical management of MIBC should result from the read out of the German and American prospective randomized trials testing extended (ePLND) versus standard (sPLND) pelvic lymph node dissection. In the meantime, Abdi et al. have added to the retrospective data suggesting a benefit to ePLND based on a matched analysis in which the decision to perform ePLND or SPLND was not based on potentially confounding patient factors, but instead strictly on individual surgeon routine practice of exclusively performing either ePLND or sPLND [7]. The authors report a lower rate of local recurrences after ePLND without a concomitant increase in perioperative complications, although the risk of blood transfusion was higher.

Systemic therapy of MIBC appears finally to be on the verge of major breakthroughs, similar to what have happened to kidney cancer a decade ago. For years MIBC has lagged behind most other cancers with respect to novel targeted therapies, but now, with rapid advances in the molecular understanding of MIBC, the field is poised for change [8]. The most immediate breakthrough will likely be the routine implementation of checkpoint inhibitors, a novel class of immunotherapy. As outlined by Kates et al., multiple agents, including especially inhibitory monoclonal antibodies that target specific immune-regulatory molecules such as PD-1, PD-L1 and CTLA-4 are under development, with 2 already reporting very promising results in the metastatic setting [9].

Beyond surgery and systemic therapy, social media is also impacting MIBC by changing how providers, patients and other stakeholders interact with each other. Leveridge outlines how social media can be utilized as a tool for patient education, patient support and dissemination of new knowledge [10]. The Patient-Centered Research Outcomes Institute (PCORI) has utilized social media and public medical communication to develop two-way learning and to canvass novel research ideas from patients. Particularly noteworthy is the potential to use social media to enhance accrual to clinical trials, which is something with which the bladder cancer community has struggled. As the younger, more technology-savvy generations mature, the influence of social media is likely to increase.

\section{References}

1. VanderWalde NA, Chi MT, Hurria A, Galsky MD, Nielsen ME (2015) Treatment of muscle invasive bladder cancer in the elderly: navigating the trade-offs of risk and benefit. World $\mathbf{J}$ Urol. doi:10.1007/s00345-015-1708-z

2. Gray PJ, Fedewa SA, Shipley WU, Efstathiou JA, Lin CC, Zietman AL, Virgo KS (2013) Use of potentially curative therapies for muscle-invasive bladder cancer in the United States: results from the National Cancer Data Base. Eur Urol 63(5):823-829. doi:10.1016/j.eururo.2012.11.015

3. Hugen CM, Daneshmand S (2015) Orthotopic urinary diversion in the elderly. World J Urol. doi:10.1007/s00345-015-1696-Z

4. Siddiqui KM, Izawa JI (2015) Ileal conduit: standard urinary diversion for elderly patients undergoing radical cystectomy. World J Urol. doi:10.1007/s00345-015-1706-1

5. Patel HRH, Santos PB, Oliveira MC, Müller S (2015) Is robotic-assisted radical cystectomy (RARC) with intracorporeal diversion becoming the new gold standard of care? World J Urol doi:10.1007/s00345-015-1730-1

6. Kiss B, Burkhard FC, Thalmann GN (2015) Open radical cystectomy: still the gold standard for muscle invasive bladder cancer. World J Urol. doi:10.1007/s00345-015-1729-7

7. Abdi H, Pourmalek F, Gleave ME, So AI, Black PC (2015) Balancing risk and benefit of extended pelvic lymph node dissection in patients undergoing radical cystectomy. World J Urol doi:10.1007/s00345-015-1734-x

8. Cancer Genome Atlas Research Network (2014) Comprehensive molecular characterization of urothelial bladder carcinoma. Nature 507:315-322

9. Kates M, Sopko NA, Matsui H, Drake CG, Hahn NM, Bivalacqua $T$ (2015) Immune checkpoint inhibitors: a new frontier in bladder cancer. World J Urol. doi:10.1007/s00345-015-1709-y

10. Leveridge MJ (2015) The state and potential of social media in bladder cancer. World J Urol. doi:10.1007/s00345-015-1725-y 